\title{
Surface quality assurance and process reliability in the processing with a ball-rod hardener
}

\author{
Mikhail Tamarkin ${ }^{1}$, Elina Tishchenko ${ }^{1,{ }^{*}}$,Elena Murugova ${ }^{1}$, and Alexandr Melnikov ${ }^{1}$ \\ ${ }^{1}$ Don State Technical University, 1, Gagarin sq., 344003, Rostov-on-Don, Russia
}

\begin{abstract}
This paper presents the results of research on the processing with a multi-contact vibratory hardening tool - ball-rod hardener. Theoretical relationships are derived for the calculation of surface roughness, the depth of the hardened layer, and the degree of deformation. The formula for determining the processing time is identified. The technological process reliability with a ball-rod hardener is investigated. Methodology of the process design with regard to its reliability assurance is presented.
\end{abstract}

\section{Introduction}

The performance properties of the part are formed throughout all stages of manufacturing process, especially during finishing operations. With the help of processing parts methods of surface plastic deformation, which are widely used in mechanical engineering, it is possible to bring vast improvement to the performance properties. Moreover, from an economic point of view, the most profitable is the local hardening of parts with stress concentrators in comparison with the bulk processing of all surfaces.

It is possible to process individual surfaces of machine parts with the surface plastic deformation methods using compact devices, one of which is a ball-rod hardener. The ballrod hardener is a universal device that allows to process both flat surfaces and surfaces of irregular form, including those with a small difference in height. The ball-rod hardener device was invented at the Technology of Mechanical Engineering Department of DSTU under the guidance of Professor A. P. Babichev [1-15].

The ball-rod hardener is quite simple in design. It contains a power drive with a striker, as well as a package of round rods installed in a special collet fixture. Several layers of steel balls are located in the enclosed volume between the rods and the drive. Pneumatic and electric grinders, which are widely used in industry, are utilized as a power drive.

The striking energy of the drive is transferred to the rod package without significant losses using steel balls. In this case, the spherical sharpening rods copy the surface shape of the part and form a plastic imprint.

The main technological parameters of the process with the ball-rod hardener include the impact energy of the striker, the diameter and number of rods, the radius of the spherical

\footnotetext{
* Corresponding author: lina tishenko@mail.ru
} 
sharpening of the rods and the tension during processing. The advantages of the ball-rod hardener processing in comparison with other shock processing methods can be attributed to the high intensity of processing, the formation of residual compression stresses and the possibility of local hardening.

Initially, this ball-rod hardener was used as a manual processing tool for sections of large-sized parts, as well as surfaces of pre-assembled parts. At the same time, there were difficulties in maintaining the movement accuracy, tension, and ensuring the required number of strokes. However, even manually, the ball-rod hardener allows to smooth out cavities, harden the surface layer and create compressive residual stresses. Nowadays, the ball-rod hardener is frequently fixed on a lathe or milling machine.

\section{Materials and Methods}

Furthermore, there is an urgent necessity to carry out theoretical research and determine the influence of technological modes on the quality of the treated surface for further processing with a ball-rod hardener. The purpose of the research is to improve the efficiency and reliability of the process, taking into account the study of the accuracy indicators. It is necessary to develop a set of adequate theoretical models describing the surface quality development during processing. Nowadays, the following relations have been identified [19]. They allow to define:

- surface roughness:

- depth of the hardened layer:

D

- degree of deformation:

- processing time:

$u$ - striking energy, $\eta$ - output of the ball-rod hardener, $M$ - number of rods in the package, $H B$ - hardness of the part material in compliance with Brinell, $F$ - number of the recurrent max-maximum depth of the indenter introduction into the part surface, $R$ - radius of the $s$ - volume of the deformed metal in a unit input

The above formulas have been tested during complex experimental studies of the ballrod hardener process.

The reinforcing device for ball-rod hardener processing was fixed on a milling or lathe. The KMP-14M pneumatic hammer was used. The Samples were made of various materials (aluminum alloys D16, B95, AVT, as well as St3, 45, and HVG steels), which are widely used in mechanical engineering and have various properties. This is necessary for the universality evaluation of the obtained theoretical models.

The radius of the spherical rods sharpening varied, and different number of rods was placed in the package. Some results of theoretical data comparison and experimental data are shown in Fig. 1 and 2. Theoretical relations are shown as solid lines on the graphs. Dots indicate the results of experiments with confidential intervals (the confidential probability is $95 \%)$. 


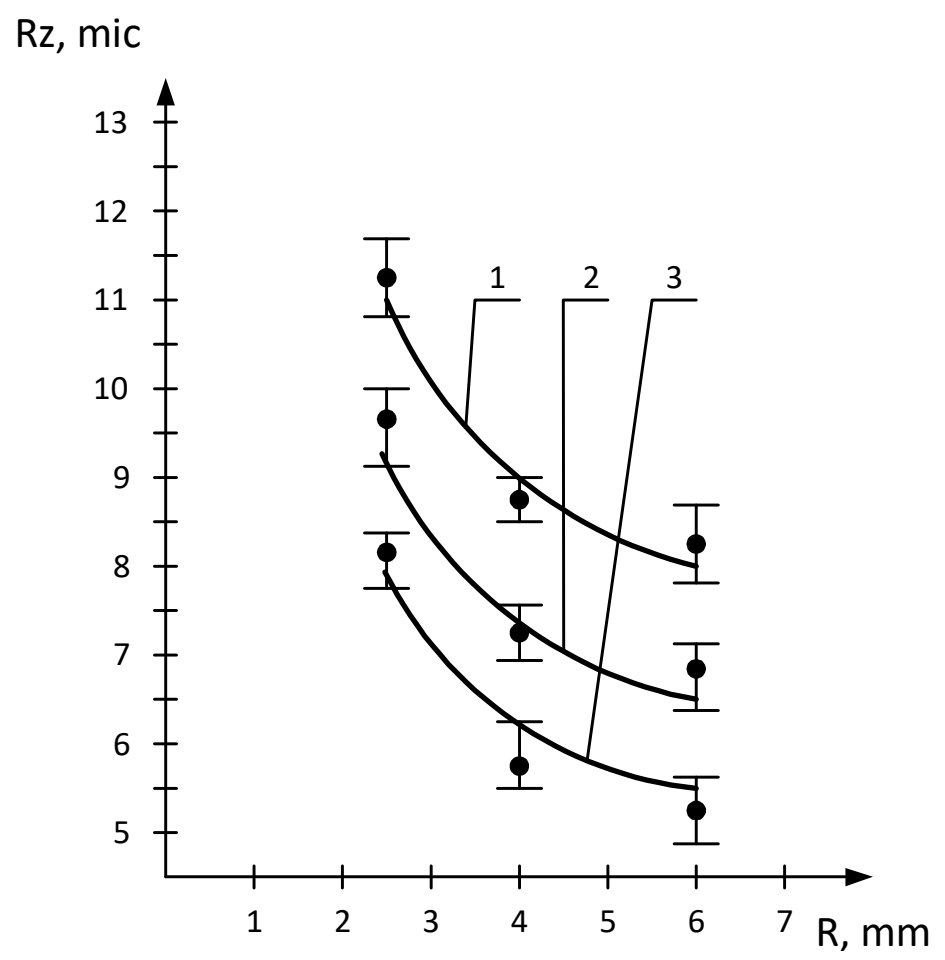

Fig. 1. The dependence of the treated surface roughness on the radius of the spherical sharpening $(\mathrm{M}=19,=0.7)$ for materials: 1-D16, 2-B95, 3 - 45 steel.

$\mathrm{hn}, \mathrm{mm}$

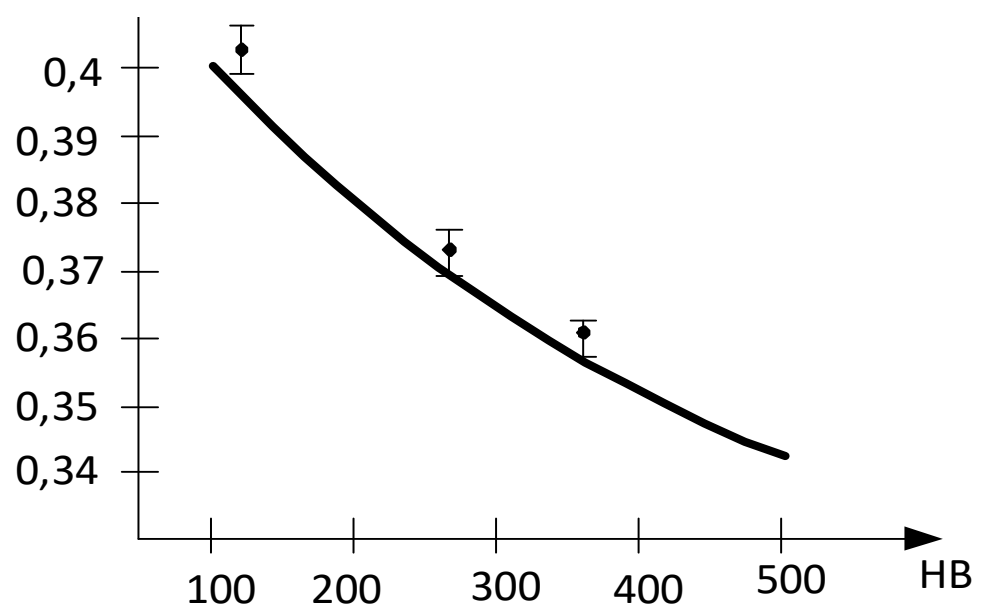

Fig. 2. Dependence of the hardened layer depth on the Brinell hardness (number of rods $M=19$, striking energy $\mathrm{Eu}=2.5 \mathrm{~J}, \eta=0.7$, radius of spherical sharpening $-3 \mathrm{~mm}$ ).

The analysis of the above data showed that the discrepancy between the theoretical and experimental values does not exceed 15\%. Thus, the proposed theoretical relations are adequate on the basis of Fisher's variance ratio and can be recommended for calculations in technological design.

The study of reliability parameters in the ball-rod hardener processing is an important aspect of the successful technological process design, which contributes to the specified 
quality of products. For reliability analysis, according to [4-6], reliability coefficients are used according to Russian Standard GOST 27.202-83. When controlling technological processes on a quantitative basis, using the relations presented in the Russian Standard, the values of accuracy indicators for the controlled parameter are determined: the accuracy $z(\tau)$ should be greater than zero.

Accuracy indicators calculations are necessary for the analytical forecasting of the reliability level of the ball-rod hardener processing. Reliability analysis allows to ensure $\operatorname{sm}(\tau)$ were calculated within possible variations of the mechanical material properties and $z(\tau)$ is defined that must be greater than zero. In this case, the process is reliable. Some calculation results are shown in Fig. 3 and Fig. 4.

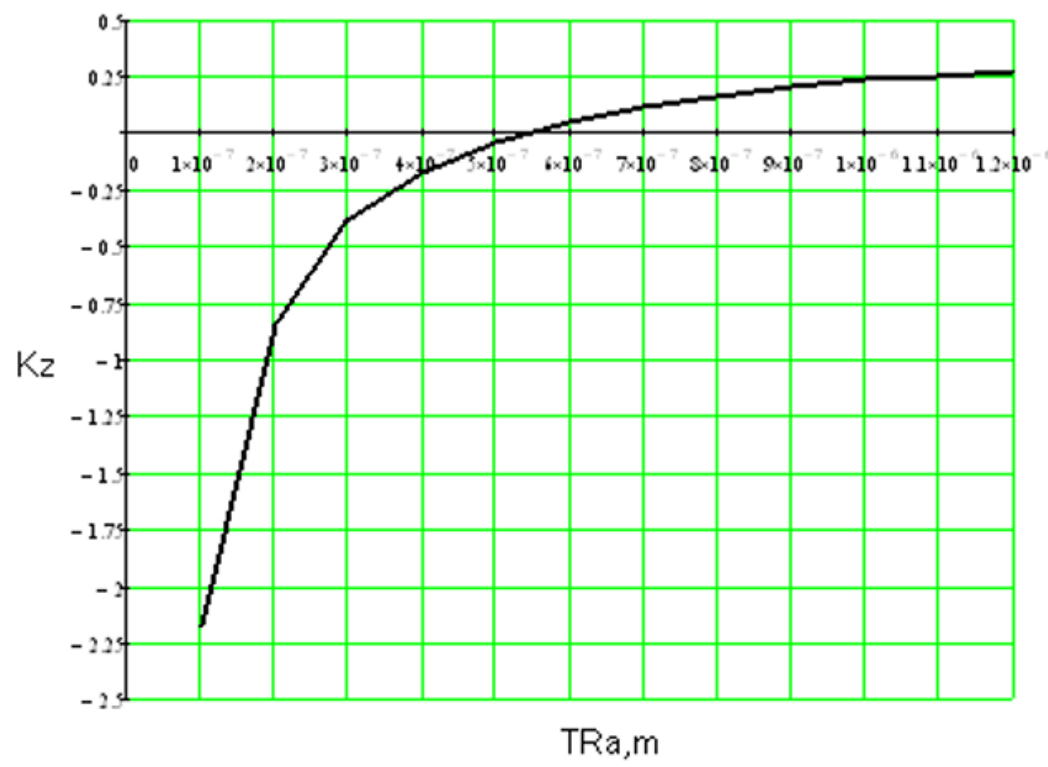

Fig. 3. Dependence of the accuracy margin coefficient on the tolerance capability of the controlled parameter Ra. Part material-45 steel, processing modes: $M=19, D=5 \mathrm{~mm}$. Spread of technological parameters-5\%. 


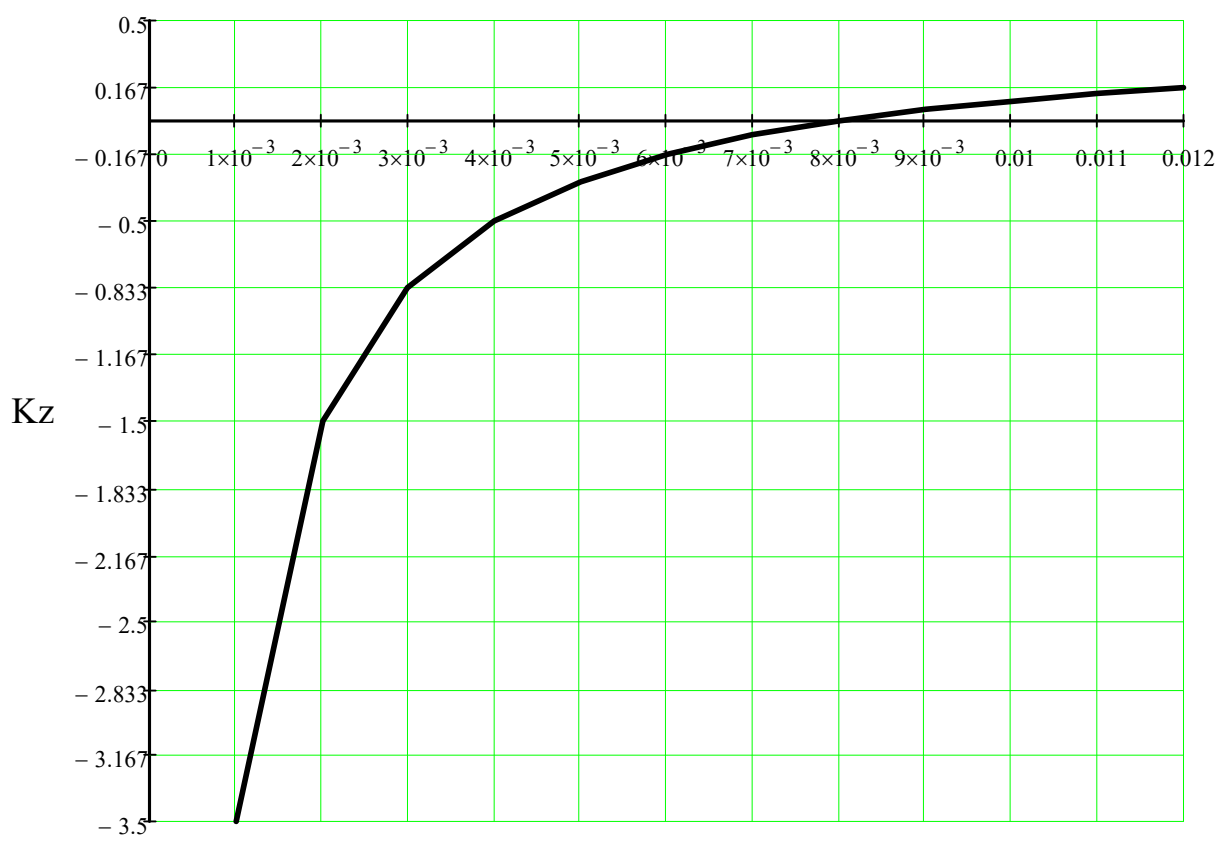

$\mathrm{T} \varepsilon$

Fig. 4. Dependence of the accuracy margin coefficient on the tolerance capability of the controlled parameter $\varepsilon$. Part material - HVG, processing modes: $M=40, D=8 \mathrm{~mm}$. Spread of technological parameters- $10 \%$.

\section{Results}

It is revealed, that technological process makes it possible to ensure the reliability of obtaining the specified quality of the surface layer of the processed parts in the range of technological parameters set for each case.

The above set of theoretical relations, which has passed the experimental test, can be used for analytical forecasting of ensuring the technological processes reliability. On the basis of the given theoretical models, methodology of designing technological processes with regard to ensuring their reliability and CAD elements in the form of software module is developed. Using the proposed software package, you can calculate the surface layer quality indicators using the formulas (1) - (3) given above.

The user enters the stray field of the original variables (technological regimes and physico-mechanical properties of the material), defines the stray fields values of a controlled parameter, the average deviation value of a controlled parameter in relation to the middle of the tolerance bands.

Furthermore, the value of the accuracy margin coefficient based on the relation $z(\tau)>0$, the processing time is calculated as per the formula (4) for each combination of technological parameters. The option that provides the minimum processing time is selected.

The obtained methodology of technological process design with the usage of ball-rod hardener will ensure its reliability. Its approbation was in production conditions and used in the implementation of the ball-rod hardener parts processing at LLC "Donkuzlitmash" in Azov city. 


\section{References}

1. M. Tamarkin, E. Tishchenko, I. Viyalikov, Russian Engineering Research 35, 740-744 (2015)

2. M. Tamarkin, E. Tishchenko, V. Fedorov, IOP Conference Series: Materials Science and Engineering 124(1) (2016)

3. K. Hamouda, H. Bournine, M. Tamarkin, A. Babichev, D. Saidi, H.E. Amrou, Materials Science 52(2), 216-221 (2016)

4. M. Tamarkin, E. Tishchenko, D. Kazakov, A. Isaev, Russian Engineering Research 37(4), 326-329 (2017)

5. M. Tamarkin, E. Tishchenko, D. Kazakov, Proceedings of the International Conference on Mechanical, System and Control Engineering, ICMSC 2017, 31-35 (2017)

6. M. Tamarkin, E. Tishchenko, A. Shvedova, Russian Engineering Research 38(9), 726727 (2018)

7. M. Tamarkin, V. Butenko, A. Isaev, E. Murugova, MATEC Web of Conferences 226 (2018)

8. M. Tamarkin, E. Tishchenko, A. Melnikov, E. Chernyshev, Advances in Intelligent Systems and Computing 983, 661-669 (2019)

9. M. Tamarkin, E. Tishchenko, I. Chukarina, T. Sosnitskaya, Proceedings of the VIII International Scientific Siberian Transport Forum - TransSiberia 20191 (2019)

10. A.N. Solovev, T.N. Van, M. Tamarkin, I.A. Panfilov, J.P. Wang, Physics and Mechanics of New Materials and Their Applications (PHENMA 2018), 334-336 (2018)

11. V.V. Ivanov, V.P. Smolentsev, V.A. Lebedev, Electroplating and surface treatment 27(2), 49-58 (2018)

12. S.A. Berberov, V.A. Lebedev, High technology in mechanical engineering 9(75), 3841 (2017)

13. V.A. Lebedev, V.D. Sokolov, IV Strengthening technologies and coatings 14.2(158), 54-58 (2018)

14. A.P. Shishkina, V.A. Lebedev, E.Yu. Krupenya, Bulletin of the Rybinsk State Aviation Technological Academy named after P.A. Solovyov 2(41), 132-137 (2017)

15. V.A. Lebedev, A.A. Kochubei, IV Bulletin of Tula State University. Technical science 8-2, 56-63 (2016) 